\title{
Mini-Review: GARP, a Putative Potential Molecule in Tumor Immunosuppressive Environment
}

\author{
Miao Guo', Wei Wu5 , Wei Liu'2, 3*, Fu Ren², 3\# \\ 'Department of Clinical Laboratory, the First Affiliated Hospital of Jinzhou Medical University, Jinzhou 121001, Liaoning, China \\ ${ }^{2}$ Biological Anthropology Institute, Jinzhou Medical University, No.40, Section 3, Songpo Road, Linghe District, Jinzhou 121001 , Liaoning, China \\ ${ }^{3}$ Liaoning Province Key Laboratory of Chinese Physical Characteristics Research (LPKL-CPCR), Jinzhou 121001, Liaoning, China \\ ${ }^{4}$ Department of Anatomy, College of Basic Medical Sciences of Jinzhou Medical University, Jinzhou 121001, Liaoning, China \\ ${ }^{5}$ School of Humanities and Management, Jinzhou Medical University, Jinzhou, Liaoning, China 121001
}

\section{Article Info}

\section{Article Notes}

Received: December 20, 2018

Accepted: January 30, 2019

\section{${ }^{*}$ Correspondence:}

Dr. Wei Liu, Biological Anthropology Institute, Jinzhou Medical University, No.40, Section 3, Songpo Road, Linghe District, Jinzhou, Liaoning, P. R. 121001, China; E-mail: dliu2018@yahoo.com.

\section{Correspondence:}

Dr. Fu Ren, Biological Anthropology Institute, Jinzhou Medical University, No.40, Section 3, Songpo Road, Linghe District, Jinzhou, Liaoning, P. R. 121001, China; E-mail: rf@jzmu.edu.cn.

(02019 Liu W*, Ren F*. This article is distributed under the terms of the Creative Commons Attribution 4.0 International License

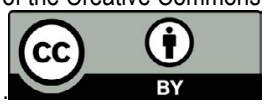

\section{Key words:}

GARP

Foxp3

Tregs

Tumor

Cancer

\section{ABSTRACT}

Glycoprotein A Repetitions Predominant (GARP), also known as leucine-rich repeats containing 32 (LRRC32), is a transmembrane protein that presents latent TGF- $\beta 1$ on the surface of regulatory T cells (Tregs) and modulates its activation in tumor immunosuppressive environment. Tregs are immunosuppressive immune cells that play an important role in tumor development and progression. Inhibition of Treg function is considered to be an effective strategy for antitumor therapy. In addition to its expression in Tregs, GARP has been recently found to be highly expressed in a few types of human solid tumor tissues, yet the role of its expression in tumor tissues or cells remains unknown. Most previous studies on GARP have focused on GARP function in Tregs and the role of GARP in latent TGF- $\beta 1$ activation. The present review provides an up to date overview of GARP expression and its potential role in tumor cells and tissues.

\section{Introduction}

Regulatory $\mathrm{T}$ cells (Tregs) are a small population of CD4+ T lymphocytes and play an important role in tumor development and progression ${ }^{1}$. Activation or enhancement of Treg function is one of the main mechanisms by which cancer evades antitumor immune attacks through a variety of different pathways. Inhibition of Treg function is also considered to be an effective antitumor treatment strategy. Previous studies have shown a significant association of expression of Treg specific markers (Foxp3, and CD39) with lymph node metastasis and poor prognosis ${ }^{2-4}$. However further studies of Tregs is limited by a of lack of available markers for activated Tregs. Although Foxp3 is very important in the development and function of Tregs ${ }^{5}$, it is expressed intracellularly in both Tregs and conventional $\mathrm{T}$ cell $\mathrm{s}^{6-8}$, making it challenging to isolate and further characterize Tregs. Specific markers for activated Tregs are therefore necessary for research in the specific mechanisms of Tregs. Glycoprotein A Repetitions Predominant (GARP), also known as leucine-rich repeats containing 32 (LRRC32), has been widely accepted to be a marker of activated Tregs, which contribute to an immunosuppressive tumor microenvironment ${ }^{9,10}$. It has been demonstrated that GARP is the cell surface docking receptor for latent TGF- $\beta$ and exerts its antitumor function, at least in part, by activating TGF- $\beta$ in tumor microenvironment ${ }^{11}$.

\section{TGF- $\beta$ and GARP}

Transforming growth factor- $\beta$ family members (TGF- $\beta 1$, TGF- $\beta 2$ 
and TGF- $\beta 3$ ) are pleiotropic cytokines expressed by most cells and are found in all tissues. They play a key role in cell proliferation, differentiation, migration, invasion and immune function, etc ${ }^{12}$. Dysregulations in TGF- $\beta$ function are associated with a variety of pathological conditions including cancer. TGF- $\beta 2$ and TGF- $\beta 3$ are mainly involved in embryonic development. TGF- $\beta 1$ has been extensively studied in regulating the immune response. Biochemically, TGF- $\beta$ exists in at least four different forms: (1) freely soluble TGF- $\beta$; (2) soluble TGF- $\beta$ associated with latencyassociated peptide (LAP), known as latent TGF- $\beta$ (LTGF- $\beta$ ); (3) TGF- $\beta$-LAP-LTBP, latent TGF- $\beta$ associated with latent TGF- $\beta$-binding protein (LTBP); and (4) membraneassociated latent form of TGF- $\beta^{13,14}$. Only TGF- $\beta$ without LAP is known to be biologically active. GARP is highly expressed on the surface of activated Tregs and the overexpression of GARP increases the inhibitory function of Tregs ${ }^{15}$. GARP can bind to latent TGF- $\beta$ and promote secretion and activation of TGF- $\beta 1^{16,17}$. Additionally, GARP protein is also found to be expressed on human tumor cells where it likely mediates the accumulation and subsequent activation of latent TGF- $\beta^{11}$. By regulating innate and adaptive immune components and facilitating tumor immune evasion, GARP provides an excellent TGF- $\beta$ reservoir that plays a role in the tumor microenvironment, thereby supporting the growth and progression of cancer cells. In terms of innate immunity, TGF- $\beta$ inhibits the maturation of natural killer (NK) cells and dendritic (DC) cells ${ }^{18,19}$. Furthermore, TGF- $\beta$ impairs the adaptive antitumor immunity by directly inhibiting the clonal expansion and cytotoxicity of the CD8+ cytotoxic T cells(CTLs) ${ }^{20,21}$. Finally, TGF- $\beta$ indirectly attenuates CTLs by inducing the expression of Foxp3, which confers a regulatory and immune suppressive phenotype to $\mathrm{CD} 4+\mathrm{T}$ cells ${ }^{22}$.

\section{GARP Gene and Expression}

The GARP-encoding gene LRRC32 was firstly identified as DI1S863E in the human 11q13.5-q14 chromosomal region and defined on chromosome 7 in the region $7 \mathrm{E}$ $7 \mathrm{~F}$ in mice ${ }^{23,24}$. LRRC32 gene is frequently amplified in breast cancer ${ }^{9,10}$. It consists of two encoding exons and is expressed at two major transcripts of 4.4 and $2.8 \mathrm{~kb}$. The first exon encodes the signal peptide and nine amino acids, and the second exon encodes most of the coding region ${ }^{25}$. GARP gene is expressed in a variety of tissues including placenta, lung, kidney, heart, liver, skeletal muscle, pancreas and lymphoid tissues ${ }^{10}$. In addition, GARP gene is detected in multiple cell types such as megakaryocytes, platelets ${ }^{26,27}$, Tregs $^{15,28}$, endothelial cells ${ }^{29,30}$ and hepatic stellate cells ${ }^{31}$, as well as embryonic stem cells and fibroblasts ${ }^{32}$. Single nucleotide polymorphisms (SNP) located in the noncoding regions of human GARP are associated with higher risks of developing diseases, including certain types of cancers ${ }^{33,34}$. GARP expression is also regulated by microRNAs. MiR-142- 3p, miR-181a, miR-185, miR-24, and miR-335 are thought to bind to the 3' UTR of GARP to repress its expression ${ }^{35,36}$. Thereafter, miR-142-3p represses posttranscriptional regulation of GARP expression by Argonaute 2-associated degradation of GARP mRNA. The Argonaute protein family plays a central role in RNA silencing processes, as essential components of the RNA-induced silencing complex (RISC). Argonaute 2 binds miR-142-3p, which guides Argonaute 2 to the 3' untranslated region of GARP through sequence complementarity, which then leads to GARP mRNA cleavage or translation inhibition. Downregulation of miRNA, in turn, may be one way to induce GARP expression in Tregs.

\section{GARP Protein and Expression}

GARP is an $80 \mathrm{kD}$ transmembrane protein consisting of 662 amino acids. Its structure can be divided into three regions: the extracellular domain with leucine-rich repeats, accounting for about $70 \%$ of the protein; the hydrophobic transmembrane domain; and cytoplasmic tail of 15 amino acid residues ${ }^{10,25}$. Tregs frequently accumulate in the tumor tissues and peripheral blood of patients with cancer. Their increased frequency has generally been considered to be the marker of poor cancer prognosis, presumably due to the Treg-mediated antitumour immunosuppression ${ }^{1}$. GARP is co-expressed with latent TGF- $\beta$ on the surface of activated Tregs, but not on other types of $\mathrm{T}$ cells and is thus regarded as a specific marker of activated Tregs ${ }^{16,37,38}$. In one study, as a TGF- $\beta$ docking receptor, the number of GARP+Foxp3+ Tregs was found to be significantly higher in patients with advanced hepatocellular carcinoma than in the control group. Furthermore, the GARP expression levels of Foxp3+ Tregs were elevated in these patients ${ }^{39}$. Compared with the control group, the expression of GARP in Tregs was increased in tumor tissues of lung cancer patients and was associated with lymph node metastasis, distant metastasis and clinical stage ${ }^{40}$. These study findings indicate that increased expression of GARP promotes cancer progression through activation of immunosuppressive functions of Tregs. More interestingly, in a clinical study of advanced gastric cancer, the tumor tissue infiltration of Foxp3+ and GARP+Tregs in the neoadjuvant chemotherapy group was significantly lower than that of the control group ${ }^{41}$. Monoclonal antibodies against GARP in GARP/TGF- $\beta 1$ complexes not only blocked the production of active TGF- $\beta 1$ by human Tregs but also inhibited the immunosuppressive activity of human Tregs in a mouse model of Xenogeneic graft -versus-host disease $^{42}$. One study however reported that only deletion of GARP in mouse Tregs was not sufficient to impair their immunosuppressive function in vivo ${ }^{43}$.

\section{GARP Protein in Cancer Tissues and Cancerous Cell Lines}

As a novel marker of activated Tregs, GARP has been 
extensively studied; most of studies were focused on Tregs from peripheral blood or cancerous tissues as noted above. Increased GARP protein expression has only been found in a few types of cancers, such as breast, lung, and colon cancers ${ }^{11}$, where the increase in GARP expression was significantly correlated with advanced stage and poor prognosis. Enforced expression of GARP in normal murine mammary cells upregulated TGF- $\beta$ bioactivity and drove oncogenesis. More importantly, treatment of mice transplanted with a murine tumor expressing GARP with anti-GARP antibody decreased the number of Tregs in the blood, and inhibited lung metastasis. Concomitant treatment of chemotherapy and anti-GARP antibody inhibited primary tumor growth. While these results demonstrate the promising function of GARP in tumor treatment, the role or function of GARP expressed by tumor cells is still unclear. Little has been reported about the role of GARP expressed in cancer cells. Interestingly, Hahn et $\mathrm{al}^{44}$ found that GARP was not only expressed in activated Tregs but also in melanoma cells. More importantly, sGARP (soluble GARP) shed from both cell types had similar inhibitory properties which contributed to an immunosuppressive tumor environment by influencing the phenotype and function of monocyticmyeloid lineage cells, inhibiting CD4+ and CD8+ effector $\mathrm{T}$ cell functions and inducing Tregs. These findings reveal a new mechanism in which GARP expression on cancer cells may be a novel molecule that contributes to the tumor immunosuppressive environment. To continue this line of research, our research team ${ }^{45}$ recently found a significant increase in the expression of GARP in papillary thyroid carcinoma (PTC) compared with benign thyroid diseases (including nodular goiter and adenoma). Compared to the benign thyroid diseases elevated GARP expression in PTC was positively correlated with increased expression of Foxp3, which is very important for the development of Tregs. However, there was no significant association of elevated expression of GARP with lymph node metastasis in PTC. This study demonstrated the potential role of GARP in the pathogenesis of PTC. Our results suggest that GARP might be a novel antitumor therapeutic target. Moreover, our research further supports the existence of a positive feedback loop between GARP and Foxp3 (Figure 1). It has been also found GARP protein expression in some cell lines such as some melanoma cell lines ${ }^{44}$.

Both GARP and Foxp3 are important markers of Tregs, but the relationship between them is still controversial. Some studies have shown that GARP and Foxp3 may form a positive feedback loop. Retroviral overexpression of GARP in antigen-specific Th cells resulted in an efficient and stable re-programming of effector $\mathrm{T}$ cells towards Tregs, which involved up-regulation of Foxp3. Lentiviral downregulation of GARP in Tregs significantly impaired the suppressor function and was associated with downregulation of Foxp3. Moreover, the downregulation of Foxp3 resulted

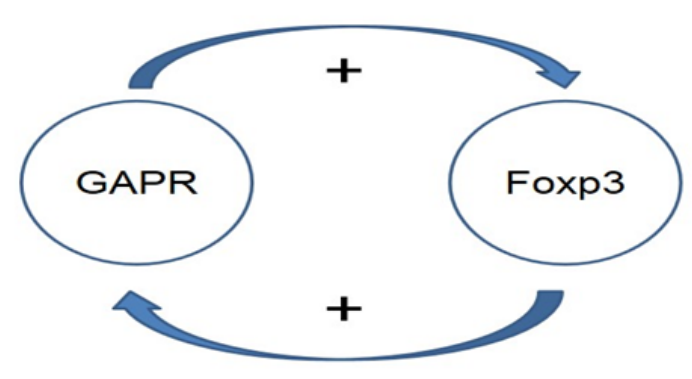

Figure 1: GARP and Foxp3 forms a positive feedback loop. Some studies have shown that increased GARP leads to an increase in Foxp3, and an increase in Foxp3 also leads to an increase in GARP, vice versa.

in similar phenotypic changes and down-regulation of GARP ${ }^{37,46}$. GARP overexpression in naïve $\mathrm{T}$ cells induced the expression of Foxp3. Silencing Foxp3 in human Tregs reduced expression of $\mathrm{GARP}^{15}$. In our study, the positive correlation between increased GARP and elevated Foxp3 expression provides further evidence for the existence of this loop ${ }^{45}$. In contrast, there are some studies that did not support the existence of a positive feedback loop between GARP and Foxp $3^{16,47,48}$. However, further studies are needed to explore the relationship between GARP and Foxp3.

\section{Conclusion and Future Perspectives}

GARP is a docking receptor for latent TGF- $\beta$ and is involved in its activation. The function of GARP-TGF- $\beta$ in Treg biology has been a topic of increasing interest. More recently, the tolerogenic role of GARP expression by cancer cells has gained attention due to the possibility that GARP could be an attractive target for antitumor immunotherapy. Moreover, since GARP is not only expressed by activated Tregs but also by some types of tumor cells, it may be an ideal target for a combinatory tumor immune therapy and serve as a novel checkpoint in future therapeutic strategies.

\section{Acknowledgment}

This study was supported by the high talent support program project of Jinzhou Medical University (2015RC011).

\section{References}

1. Frydrychowicz M, Boruczkowski M, Kolecka-Bednarczyk A, et al. The Dual Role of Treg in Cancer. Scand J Immunol. 2017; 86: 436-443.

2. Aliagas E, Vidal A, Texidó L, et al. High expression of ecto-nucleotidases CD39 and CD73 in human endometrial tumors. Mediators Inflamm. 2014; $2014: 509027$.

3. Ruiz-Arguelles GJ, San Miguel JF. Cell surface markers in multiple myeloma. Mayo Clin Proc. 1994; 69: 684-690.

4. Ryzhov SV, Pickup MW, Chytil A, et al. Role of TGF-beta signaling in generation of CD39+CD73+ myeloid cells in tumors. J Immunol. 2014; 193: 3155-3164.

5. Hori S, Nomura T, Sakaguchi S. Control of regulatory T cell development by the transcription factor Foxp3. Science. 2003; 299: 1057-1061. 
6. Gavin MA, Torgerson TR, Houston E, et al. Single-cell analysis of normal and FOXP3-mutant human T cells: FOXP3 expression without regulatory T cell development. Proc Natl Acad Sci U S A. 2006; 103: 6659-6664.

7. Ramsdell F, Ziegler SF. FOXP3 and scurfy: how it all began. Nat Rev Immunol. 2014; 14: 343-349.

8. Tran DQ, Ramsey H, Shevach EM. Induction of FOXP3 expression in naive human CD4+FOXP3 $\mathrm{T}$ cells by T-cell receptor stimulation is transforming growth factor-beta dependent but does not confer a regulatory phenotype. Blood. 2007; 110: 2983-2990.

9. Szepetowski P, Ollendorff V, Grosgeorge J, et al. DNA amplification at 11q13.5-q14 in human breast cancer. Oncogene. 1992; 7: 2513-2517.

10. Ollendorff $V$, Noguchi T, deLapeyriere 0 , et al. The GARP gene encodes a new member of the family of leucine-rich repeat-containing proteins. Cell Growth Differ. 1994; 5: 213-219.

11. Metelli A, Wu BX, Fugle CW, et al. Surface Expression of TGFbeta Docking Receptor GARP Promotes Oncogenesis and Immune Tolerance in Breast Cancer. Cancer Res. 2016; 76: 7106-7117.

12. Miyazono K, Katsuno Y, Koinuma D, et al. Intracellular and extracellular TGF-beta signaling in cancer: some recent topics. Front Med. 2018; 12: $387-411$

13. Meng XM, Nikolic-Paterson DJ, Lan HY. TGF-beta: the master regulator of fibrosis. Nat Rev Nephrol. 2016; 12: 325-338.

14. Kanzaki T, Olofsson A, Morén A, et al. TGF-beta 1 binding protein: a component of the large latent complex of TGF-beta 1 with multiple repeat sequences. Cell. 1990; 61: 1051-1061.

15. Wang R, Wan $Q$, Kozhaya $L$, et al. Identification of a regulatory $\mathrm{T}$ cell specific cell surface molecule that mediates suppressive signals and induces Foxp3 expression. PLoS One. 2008; 3: e2705.

16. Tran DQ, Andersson J, Wang R, et al. GARP (LRRC32) is essential for the surface expression of latent TGF-beta on platelets and activated FOXP3+ regulatory T cells. Proc Natl Acad Sci U S A. 2009; 106: 13445-13450.

17. Wang R, Zhu J, Dong X, et al. GARP regulates the bioavailability and activation of TGFbeta. Mol Biol Cell. 2012; 23: 1129-1139.

18. Marcoe JP, Lim JR, Schaubert KL, et al. TGF-beta is responsible for NK cell immaturity during ontogeny and increased susceptibility to infection during mouse infancy. Nature immunology. 2012; 13: 843-850.

19. Novitskiy SV, Pickup MW, Chytil A, et al. Deletion of TGF-beta signaling in myeloid cells enhances their antitumorigenic properties. Journal of leukocyte biology. 2012; 92: 641-651.

20. Thomas DA, Massague J. TGF-beta directly targets cytotoxic T cell functions during tumor evasion of immune surveillance. Cancer cell. 2005; 8: 369-380.

21. Gorelik L, Flavell RA. Immune-mediated eradication of tumors through the blockade of transforming growth factor-beta signaling in T cells. Nature medicine. 2001; 7: 1118-1122.

22. Shevach EM. Mechanisms of foxp $3+T$ regulatory cell-mediated suppression. Immunity. 2009; 30: 636-645.

23. Ollendorff V, Szepetowski P, Mattei MG, et al. New gene in the homologous human 11q13-q14 and mouse 7F chromosomal regions. Mamm Genome. 1992; 2: 195-200.

24. Seldin MF, Saunders AM, Rochelle JM, et al. A proximal mouse chromosome 9 linkage map that further defines linkage groups homologous with segments of human chromosomes 11, 15, and 19. Genomics. 1991; 9: 678-685.

25. Stockis J, Dedobbeleer O, Lucas S. Role of GARP in the activation of latent TGF-beta1. Mol Biosyst. 2017; 13: 1925-1935.

26. Macaulay IC, Tijssen MR, Thijssen-Timmer DC, et al. Comparative gene expression profiling of in vitro differentiated megakaryocytes and erythroblasts identifies novel activatory and inhibitory platelet membrane proteins. Blood. 2007; 109: 3260-3269.

27. Roubin R, Pizette S, Ollendorff V, et al. Structure and developmental expression of mouse Garp, a gene encoding a new leucine-rich repeatcontaining protein. Int J Dev Biol. 1996; 40: 545-555.

28. Oida T, Weiner HL. TGF-beta induces surface LAP expression on murine CD4 T cells independent of Foxp3 induction. PLoS One. 2010; 5: e15523.

29. Carrillo-Galvez AB, Cobo M, Cuevas-Ocaña S, et al. Mesenchymal stromal cells express GARP/LRRC32 on their surface: effects on their biology and immunomodulatory capacity. Stem Cells. 2015; 33: 183-195.

30. Carambia A, Freund B, Schwinge D, et al. TGF-beta-dependent induction of $\mathrm{CD} 4(+) \mathrm{CD} 25(+) \mathrm{Foxp} 3(+)$ Tregs by liver sinusoidal endothelial cells. J Hepatol. 2014; 61: 594-599.

31. Li Y, Kim BG, Qian S, et al. Hepatic Stellate Cells Inhibit T Cells through Active TGF-beta1 from a Cell Surface-Bound Latent TGF-beta1/GARP Complex. J Immunol. 2015; 195: 2648-2656.

32. Kehrmann A, Truong H, Repenning A, et al. Complementation of nontumorigenicity of HPV18-positive cervical carcinoma cells involves differential mRNA expression of cellular genes including potential tumor suppressor genes on chromosome 11q13. Cancer Genet. 2013; 206: 279-292.

33. Goode EL, DeRycke M, Kalli KR, et al. Inherited variants in regulatory T cell genes and outcome of ovarian cancer. PLoS One. 2013; 8: e53903.

34. Derycke MS, Charbonneau B, Preston CC, et al. Toward understanding the genetics of regulatory $\mathrm{T}$ cells in ovarian cancer. Oncoimmunology. 2013; 2: e24535.

35. Gauthy E, Cuende J, Stockis J, et al. GARP is regulated by miRNAs and controls latent TGF-beta 1 production by human regulatory $\mathrm{T}$ cells. PLoS One. 2013; 8: e76186.

36. Zhou Q Haupt S, Prots I, et al. miR-142-3p is involved in CD25+ CD4 $\mathrm{T}$ cell proliferation by targeting the expression of glycoprotein $\mathrm{A}$ repetitions predominant. J Immunol. 2013; 190: 6579-6588.

37. Probst-Kepper M, Geffers R, Kröger A, et al. GARP: a key receptor controlling FOXP3 in human regulatory T cells. J Cell Mol Med. 2009; 13: 3343-3357.

38. Stockis J, Colau D, Coulie PG, et al. Membrane protein GARP is a receptor for latent TGF-beta on the surface of activated human Treg. Eur J Immunol. 2009; 39: 3315-3322.

39. Kalathil S, Lugade AA, Miller A, et al. Higher frequencies of GARP(+) CTLA-4(+)Foxp3(+) T regulatory cells and myeloid-derived suppressor cells in hepatocellular carcinoma patients are associated with impaired T-cell functionality. Cancer Res. 2013; 73: 2435-2444.

40. Jin H, Sun L, Tang L, et al. Expression of GARP Is Increased in TumorInfiltrating Regulatory T Cells and Is Correlated to Clinicopathology of Lung Cancer Patients. Front Immuno. 2017; 18: 138.

41. Li K, Chen F, Xie H. Decreased FOXP3+ and GARP+ Tregs to neoadjuvant chemotherapy associated with favorable prognosis in advanced gastric cancer. Onco Targets Ther. 2016; 9: 3525-3533.

42. Cuende J, Liénart S, Dedobbeleer 0, et al. Monoclonal antibodies against GARP/TGF-beta1 complexes inhibit the immunosuppressive activity of human regulatory T cells in vivo. Sci Transl Med. 2015; 7: 284ra256.

43. Vermeersch E, Liénart S, Collignon A, et al. Deletion of GARP on mouse regulatory $T$ cells is not sufficient to inhibit the growth of transplanted tumors. Cell Immunol. 2018; 332: 129-133.

44. Hahn SA, Neuhoff A, Landsberg J, et al. A key role of GARP in the immune suppressive tumor microenvironment. Oncotarget. 2016; 7 : 42996-43009. 
45. Zhang X, Guo M, Yang J, et al. Increased Expression of GARP in Papillary Thyroid Carcinoma. Endocr Pathol. 2018.

46. Probst-Kepper M, Kröger A, Garritsen HS, et al. Perspectives on Regulatory T Cell Therapies. Transfus Med Hemother. 2009; 36: 302-308.

47. Wang R, Kozhaya L, Mercer F, et al. Expression of GARP selectively identifies activated human FOXP3+ regulatory T cells. Proc Natl Acad Sci U S A. 2009; 106: 13439-13444.

48. Elkord E, Abd Al Samid M, Chaudhary B. Helios, and not FoxP3, is the marker of activated Tregs expressing GARP/LAP. Oncotarget. 2015; 6: 20026-20036. 